\title{
BROWNIAN PARTICLES WITH ELECTROSTATIC REPULSION ON THE CIRCLE: DYSON'S MODEL FOR UNITARY RANDOM MATRICES REVISITED
}

\author{
Emmanuel CÉPA $^{1}$ And Dominique LÉPINGle ${ }^{1}$
}

\begin{abstract}
The Brownian motion model introduced by Dyson [7] for the eigenvalues of unitary random matrices $N \times N$ is interpreted as a system of $N$ interacting Brownian particles on the circle with electrostatic inter-particles repulsion. The aim of this paper is to define the finite particle system in a general setting including collisions between particles. Then, we study the behaviour of this system when the number of particles $N$ goes to infinity (through the empirical measure process). We prove that a limiting measure-valued process exists and is the unique solution of a deterministic second-order PDE. The uniform law on $[-\pi ; \pi]$ is the only limiting distribution of $\mu_{t}$ when $t$ goes to infinity and $\mu_{t}$ has an analytical density.
\end{abstract}

Mathematics Subject Classification. 60K35, 60F05, 60H10, 60J60.

Received December 20, 2000. Revised October 8, 2001.

\section{INTRODUCTION}

The aim of this paper is to study the behaviour of an interacting $N$-Brownian particles system on the circle with electrostatic inter-particles repulsion, when the number of particles $N$ tends to infinity. More precisely, we are interested in the system of particles governed by

$$
\mathrm{d} X_{t}^{(j)}=\mathrm{d} B_{t}^{(j)}+\frac{\lambda}{N} \sum_{1 \leqslant k \neq j \leqslant N} \cot \left(\frac{X_{t}^{(j)}-X_{t}^{(k)}}{2}\right) \mathrm{d} t, j=1,2, \ldots, N,
$$

and then transported onto the circle by setting $Z_{t}^{(j)}=\mathrm{e}^{i X_{t}^{(j)}}, 1 \leqslant j \leqslant N$. Firstly, we have to show the existence of the solution for this system (with singular drift), and then the behaviour of the solution when $N \uparrow \infty$ is considered through the sequence of empirical measure processes

$$
\mu_{t}^{(N)}=\frac{1}{N} \sum_{j=1}^{N} \delta_{Z_{t}^{(j)}}
$$

(where $\delta_{x}$ is the Dirac probability at $x$ ) by studying the weak convergence of this sequence and identifying the limit.

Keywords and phrases: Repulsive particles, multivalued stochastic differential equations, empirical measure process.

1 MAPMO, UMR 6628, bâtiment de Mathématiques, Université d'Orléans, BP. 6759, 45067 Orléans Cedex 2, France; e-mail: cepa@labomath.univ-orleans.fr and lepingle@labomath.univ-orleans.fr 
In a previous paper [4], the same program was applied to the interacting $N$-Brownian particles system on $\mathbb{R}$ with electrostatic inter-particles repulsion

$$
\mathrm{d} X_{t}^{(j)}=\mathrm{d} B_{t}^{(j)}+\frac{2 \lambda}{N} \sum_{1 \leqslant k \neq j \leqslant N} \frac{\mathrm{d} t}{X_{t}^{(j)}-X_{t}^{(k)}}, j=1,2, \ldots, N .
$$

The empirical distributions $\left(\mu^{(N)}\right)_{N}$ were shown to converge towards the unique (deterministic) continuous probability measure-valued function $\mu$ which is (classical) solution of the so-called McKean-Vlasov equation

$$
\left\{\begin{array}{l}
\frac{\partial \mu_{t}}{\partial t}=\frac{1}{2} \frac{\partial^{2} \mu_{t}}{\partial x^{2}}-2 \lambda \frac{\partial\left(\mu_{t} \mathcal{H}\left(\mu_{t}\right)\right)}{\partial x} \\
\mu_{0}=\delta_{0}
\end{array}\right.
$$

where $\mathcal{H}\left(\mu_{t}\right)$ stands for the Hilbert transform of $\mu_{t}$ given by $\mathcal{H}(\nu)=p v\left(\frac{1}{x}\right) * \nu$. Moreover, in [1], the regularity results obtained for $\mu_{t}$ and $\mathcal{H}\left(\mu_{t}\right)$ allowed us to show existence/uniqueness of the strong solution for the non-linear SDE (in the sense of McKean) associated with (1.3) (see [23] for a general reference)

$$
\left\{\begin{array}{l}
X_{t}=B_{t}+2 \lambda \int_{0}^{t} \mathcal{H} \mu\left(s, X_{s}\right) \mathrm{d} s \\
X_{0}=0 ; X_{t} \sim \mu_{t}, t>0
\end{array}\right.
$$

where the notation " $Y \sim \nu$ " means that the random variable $Y$ has the law $\nu$.

In the present case, we will prove that the system (1.1) is well-defined for all $\lambda>0$ and give necessary/sufficient conditions for existence of collisions between particles $Z^{(j)}$. Furthermore, the limiting measure-valued process $\mu$ of $\mu^{(N)}$ exists and is the unique continuous probability measure-valued function which satisfies

$$
\begin{aligned}
\int_{-\pi}^{\pi} f(x) \mu_{t}(\mathrm{~d} x)= & f(0)+\frac{1}{2} \int_{0}^{t} \mathrm{~d} s\left(\int_{-\pi}^{\pi} f^{\prime \prime}(x) \mu_{s}(\mathrm{~d} x)\right) \\
& +\frac{\lambda}{2} \int_{0}^{t} \mathrm{~d} s\left[\int_{-\pi}^{\pi} \int_{-\pi}^{\pi}\left(f^{\prime}(x)-f^{\prime}(y)\right) \cot \left(\frac{x-y}{2}\right) \mu_{s}(\mathrm{~d} x) \mu_{s}(\mathrm{~d} y)\right]
\end{aligned}
$$

for all $2 \pi$-periodic $C^{2}$ functions $f$. In other words, $\mu$ is the unique weak solution of

$$
\left\{\begin{array}{l}
\frac{\partial \mu_{t}}{\partial t}=\frac{1}{2} \frac{\partial^{2} \mu_{t}}{\partial x^{2}}-2 \lambda \frac{\partial\left(\mu_{t} \mathbb{H}\left(\mu_{t}\right)\right)}{\partial x} \\
\mu_{0}=\delta_{0}
\end{array}\right.
$$

where $\mathbb{H}\left(\mu_{t}\right)$ is the periodic Hilbert transform of $\mu_{t}$ given by $\mathbb{H}(\nu)=\frac{1}{2} p v\left(\cot \left(\frac{x}{2}\right)\right) * \nu$.

If one thinks of the particles $Z^{(j)}$ on the circle, then the system (1.1) can be interpreted as a system of particles driven by a Brownian motion, constrained to stay on the circle, and such that each pair of particles interacts via a repulsion force proportional to the inverse distance between them. Note that each particle interacts with all the others and because of the explosion of $\cot (. / 2)$ at 0 and $2 \pi$, all particles are mutually repelling: in particular, the "last" one and the "first" one interact through an electrostatic repulsion as well. We can notice that $\left|2 \sin \left(\frac{x-y}{2}\right)\right|=\left|\mathrm{e}^{i x}-\mathrm{e}^{i y}\right|$ and that the cotangent factor is a result of projecting the force onto the tangent to the circle at $\mathrm{e}^{i x}$. In [15], Metivier suggested the problem of studying the behaviour of $N$-particles system when $N \uparrow \infty$ for an interaction exploding like $1 / x$ near 0 . Indeed, this situation was not covered by the papers on this subject which use Lipschitz/boundedness conditions in the drift term. In [16], a class of singular drift is 
considered with a different setting: a boundary (local time) term exists in the non-linear SDE and the approach is based on the Skorohod problem. The natural and physical interpretation of systems like (1.1) and (1.3) as systems of Brownian particles submitted to an electrostatic repulsion and living on $\mathbb{R}$ or on the circle is the first obvious motivation. One can give other reasons making this kind of singular particle system of particular interest.

Let us begin with the main motivation of the systems (1.1) and (1.3), or the same equation as in (1.3) with the interaction $1 / x$ being replaced by the interaction $\operatorname{coth}(x)$ as well. In fact, these systems have much to do with the eigenvalues of random matrices (see [14] for a general reference on the subject). In his pioneering work, Dyson [7] proposed Brownian models for these eigenvalues. More precisely, if one considers a random symmetric $N \times N$ matrix whose coefficients are independent Brownian motions, then the eigenvalues perform a diffusion satisfying an equation of the type (1.3). A sketch of this situation is given in [13] (p. 124). In [5] and [19], Brownian motions are replaced by Ornstein-Uhlenbeck processes: the authors observe the asymptotic behaviour as $N$ goes to infinity and the semi-circle Wigner law emerges somewhere as a limit distribution, which is consistent with the interpretation of random matrices in terms of free probability theory and the free central limit theorem: see [25]. On the other hand, if one considers random unitary matrices with eigenvalues $Z^{(j)}=\mathrm{e}^{i X^{(j)}}$, then the $X^{(j)}$ 's satisfy a system of the class (1.1). In order to be complete, let us point out that if $\left\{G_{t}\right\}$ is a right-invariant Brownian motion on $S L(2 ; \mathbb{R})$ and if $\mathrm{e}^{U_{t}}, \mathrm{e}^{-U_{t}}$ denote the eigenvalues of $G_{t}^{T} G_{t}$, the process $U_{t}$ is a solution of the equation

$$
\mathrm{d} U_{t}=\sqrt{2} \mathrm{~d} B_{t}+\operatorname{coth}\left(U_{t}\right) \mathrm{d} t
$$

The reader can consult [20] (p. 241) for these links as well as other motivations for singular systems from stochastic differential geometry: Dynkin's Brownian motion of ellipses, Brownian motion on Lie groups, ...

An extension of Dyson's model has recently been developed by Grabiner [9]: he considers a Brownian motion conditioned never to exit the Weyl chamber of a semisimple Lie algebra. When the Weyl chamber is given by the conditions $x^{(1)}<x^{(2)}<\cdots<x^{(N)}$, he finds again Dyson's model of $N$ particles moving in a diffusion with a repelling force proportional to the inverse of the distance between two particles. As a matter of fact, singular diffusions like (1.1) or (1.3) have strong connections with conditioned processes and $h$-processes. For example, it is shown in [17] that the process in (1.1) with the term $\lambda / N$ equal to $1 / 2$ corresponds to a system of $N$ Brownian particles conditioned never to collide. More precisely, this result means that the law of $\left(B_{t \wedge M}, t \geqslant 0\right)$ conditional to $T>M$ ( $T$ is the first collision time) converges towards the law of the process $X$ in (1.1) when $M \rightarrow \infty$.

In [10], the probability transition density for $N$ Brownian motions on the circle killed when any pair collide is calculated. The problem is therefore linked with the exit time of the domain

$$
D_{N}=\left\{x=\left(x^{(1)}, x^{(2)}, \ldots, x^{(N)}\right) \in \mathbb{R}^{N}: x^{(1)}<x^{(2)}<\cdots<x^{(N)}<x^{(1)}+2 \pi\right\},
$$

by a $N$-dimensional Brownian motion: this problem may also be interpreted in terms of eigenvalues/eigenfunctions of the Laplacian in $D_{N}$ with Dirichlet boundary conditions.

Let us notice that one of the motivations of [10] was to help develop an intuition for the behaviour of non-intersection exponents for $N$ Brownian motions in the plane as $N \rightarrow \infty$ : see [10] and [6] for this analogy.

Finally, we point out that in [22], the behaviour of (1.1) when $N \rightarrow \infty$ is considered under the assumption of stationarity.

We will give in the last section some results without proof for the case when the interaction cot in (1.1) is replaced by coth.

As a conclusion, the stochastic differential system

$$
\mathrm{d} X_{t}^{(j)}=\mathrm{d} B_{t}^{(j)}+\frac{\gamma}{2} \sum_{1 \leqslant k \neq j \leqslant N} \cot \left(\frac{X_{t}^{(j)}-X_{t}^{(k)}}{2}\right) \mathrm{d} t, j=1,2, \ldots, N
$$


is proved to be well-defined for all $\gamma>0$ and general initial conditions, which is necessary to study the behaviour of this singular particle system when the number $N$ of particles tends to infinity and for the normalization $\gamma \sim 1 / N$. Let us notice that one has to deal with collisions in this equation because collisions happen if and only if $\gamma<1 / 2$; for $\gamma \sim 1 / N$, there are "more and more" collisions. Among the asymptotic results, a limiting non-linear PDE on the torus is obtained and studied for the empirical measure processes. The results proved in [1] and [4] are still valid in the present periodic situation (particularly, the same holomorphic Burgers equation appears at the end of the paper); in addition, the convergence of $\mu_{t}$ towards a limit distribution is an easy consequence of the compactness of the state space. Furthermore, the results allow us to wait for a convergence at the level of processes when $N \uparrow \infty$ (propagation of chaos) and the possibility of a non-linear SDE on the torus. For the reader's convenience, the proofs are (essentially) completely given even if some ideas are not new as previously quoted.

\section{Stochastic VARIATIONAL INEQUALities REVISITED}

The question of stochastic variational inequalities (SVI) is part of the general theory of multivalued SDE's. For the reader's convenience, we recall basic facts on SVI's in a simplified but classical situation. The reader will find in [2] a probabilistic approach to SVI, in [3] a deterministic one and [4] gives a representation of the solution which is applied to the system of real Brownian particles with electrostatic repulsion.

\subsection{Assumptions}

$* N \in \mathbb{N}^{*}$;

* $D$ convex non-empty open set in $\mathbb{R}^{N}$

$\left.\left.* \Phi: \mathbb{R}^{N} \rightarrow\right]-\infty ;+\infty\right]$ a l.s.c. convex function on $\mathbb{R}^{N}$ such that $D=\{\Phi<+\infty\}$ and $\Phi$ is $C^{1}$ in $D$;

$* g: \mathbb{R}^{N} \rightarrow \mathbb{R}^{N}$ and $\sigma: \mathbb{R}^{N} \rightarrow \mathbb{R}^{N} \otimes \mathbb{R}^{N}$ satisfy a global Lipschitz condition;

$* x_{0} \in \bar{D}$.

The next lemma was very useful in [2] in order to obtain existence results for SVI's.

Lemma 2.1. For every $a \in D$, there exist $\alpha>0, \beta>0$ and $\delta>0$ such that for all $x \in D$ :

$$
\langle\nabla \Phi(x), x-a\rangle \geqslant \alpha|\nabla \Phi(x)|-\beta|x-a|-\delta .
$$

Example 1. Set $F=\bar{D}$ and define

$$
\Phi_{r}(x)= \begin{cases}0 & \text { if } x \in F \\ +\infty & \text { if } x \notin F\end{cases}
$$

Remark. In fact, this example is not a good one since the domain of the convex function is not open in this case. Nevertheless, we keep it in mind as a reference.

Other interesting examples of convex function are provided by the following construction where the basic ingredient is a convex function on $\mathbb{R}$.

Proposition 2.2. Let $0<M \leqslant \infty, I=] 0 ; M[, \phi: \mathbb{R} \rightarrow]-\infty ;+\infty]$ a convex function on $I$ such that $\phi=+\infty$ in $\mathbb{R} \backslash I, \phi$ is $C^{1}$ in $I$ and $\phi\left(0^{+}\right)=+\infty, \phi\left(M^{-}\right)=+\infty$ if $M<\infty$. We define a new function on $\mathbb{R}^{N}(N \geqslant 2)$ by

$$
\Phi(x)= \begin{cases}\sum_{\substack{1 \leqslant j<k \leqslant N \\+\infty}} \phi\left(x^{(k)}-x^{(j)}\right) & \text { if } x^{(1)}<x^{(2)}<\cdots<x^{(N)}<x^{(1)}+M \\ & \text { if not }\end{cases}
$$


where $x=\left(x^{(1)}, x^{(2)}, \ldots, x^{(N)}\right)$. Then $\Phi$ is a l.s.c. convex function on $\mathbb{R}^{N}$ satisfying the previous hypothesis with the open domain

$$
D=\left\{x=\left(x^{(1)}, x^{(2)}, \ldots, x^{(N)}\right) \in \mathbb{R}^{N}: x^{(1)}<x^{(2)}<\cdots<x^{(N)}<x^{(1)}+M\right\} .
$$

Moreover, the inequality (2.1) can be improved to give (in the same context as before) for any $a \in D$

$$
\langle\nabla \Phi(x), x-a\rangle \geqslant \alpha \sum_{j<k}\left|\phi^{\prime}\left(x^{(k)}-x^{(j)}\right)\right|-\beta|x-a|-\delta .
$$

Proof. Inequality (2.5) is obtained by several applications of (2.1) to $\phi$, a sum of the inequalities over the indices $(j, k)$ and a good choice of the constants. Simple calculations show that:

$$
\begin{aligned}
\langle\nabla \Phi(x), x-a\rangle & =\sum_{j<k} \sum_{j} \phi^{\prime}\left(x^{(k)}-x^{(j)}\right)\left(x^{(k)}-a^{(k)}\right)-\sum_{j>k} \phi^{\prime}\left(x^{(j)}-x^{(k)}\right)\left(x^{(k)}-a^{(k)}\right) \\
& =\sum_{j<k} \sum_{j} \phi^{\prime}\left(x^{(k)}-x^{(j)}\right)\left[\left(x^{(k)}-x^{(j)}\right)-\left(a^{(k)}-a^{(j)}\right)\right]
\end{aligned}
$$

and, using (2.1)

$$
\begin{aligned}
\phi^{\prime}\left(x^{(k)}-x^{(j)}\right)\left[\left(x^{(k)}-x^{(j)}\right)-\left(a^{(k)}-a^{(j)}\right)\right] \geqslant & \alpha_{j, k}\left|\phi^{\prime}\left(x^{(k)}-x^{(j)}\right)\right| \\
& -\beta_{j, k}\left|\left(x^{(k)}-x^{(j)}\right)-\left(a^{(k)}-a^{(j)}\right)\right|-\delta_{j, k}
\end{aligned}
$$

and the optimization over the finite families $\left(\alpha_{j, k}\right),\left(\beta_{j, k}\right)$ finishes the proof.

The three following examples will be our references below:

Example 2. Let $d>1$ and

$$
\phi_{b}(x)= \begin{cases}-\frac{d-1}{2} \ln (x) & \text { if } x>0 \\ +\infty & \text { if } x \leqslant 0\end{cases}
$$

We set $\gamma=\frac{d-1}{2}>0$. The associated function $\Phi_{b}$ on $\mathbb{R}^{N}$ is:

$$
\Phi_{b}(x)= \begin{cases}-\gamma \sum_{1 \leqslant j<k \leqslant N} \ln \left(x^{(k)}-x^{(j)}\right) & \text { if } x^{(1)}<x^{(2)}<\cdots<x^{(N)} \\ +\infty & \text { if not. }\end{cases}
$$

Example 3. Let $\gamma>0$ and

$$
\phi_{l}(x)= \begin{cases}-\gamma \ln \left[\sin \left(\frac{x}{2}\right)\right] & \text { if } 0<x<2 \pi \\ +\infty & \text { if not. }\end{cases}
$$

The associated function $\Phi_{l}$ on $\mathbb{R}^{N}$ is:

$$
\Phi_{l}(x)= \begin{cases}-\gamma \sum_{1 \leqslant j<k \leqslant N} \ln \left[\sin \left(\frac{x^{(k)}-x^{(j)}}{2}\right)\right] & \text { if } x^{(1)}<x^{(2)}<\cdots<x^{(N)}<x^{(1)}+2 \pi \\ & \text { if not. }\end{cases}
$$


Example 4. Let $\gamma>0$ and

$$
\phi_{h}(x)= \begin{cases}-\gamma \ln [\sinh (x)] & \text { if } x>0 \\ +\infty & \text { if not. }\end{cases}
$$

The associated function $\Phi_{h}$ on $\mathbb{R}^{N}$ is:

$$
\Phi_{h}(x)= \begin{cases}-\gamma \sum_{1 \leqslant j<k \leqslant N} \ln \left[\sinh \left(x^{(k)}-x^{(j)}\right)\right] & \text { if } x^{(1)}<x^{(2)}<\cdots<x^{(N)} \\ +\infty & \text { if not. }\end{cases}
$$

\subsection{Problem}

We take $\left(\Omega, \mathcal{F},\left\{\mathcal{F}_{t}\right\}, \mathbb{P}\right)$ a filtered probability space satisfying the usual conditions, $B=\left\{B_{t}, \mathcal{F}_{t} ; 0 \leqslant t<\infty\right\}$ a N-dimensional Brownian motion on $(\Omega, \mathcal{F}, \mathbb{P})$ with $B_{0}=0$. Then, we are looking for a continuous adapted process $X=\left\{X_{t}, \mathcal{F}_{t} ; 0 \leqslant t<\infty\right\}$ whose values belong to $\bar{D}$ with $X_{0}=x_{0}$ and a continuous real increasing adapted process $L=\left\{L_{t}, \mathcal{F}_{t} ; 0 \leqslant t<\infty\right\}$ with $L_{0}=0$, such that

$$
\mathrm{d} X_{t}=g\left(X_{t}\right) \mathrm{d} t+\sigma\left(X_{t}\right) \mathrm{d} B_{t}-\nabla \Phi\left(X_{t}\right) \mathrm{d} t-n\left(X_{t}\right) \mathrm{d} L_{t} ; \quad 0 \leqslant t<\infty
$$

with $n(x) \in \pi(x)(\pi(x)$ : set of unitary outward normals to $D$ at $x), L$ is a boundary process

$$
L_{t}=\int_{0}^{t} \mathbb{I}_{\left\{X_{s} \in \partial D\right\}} \mathrm{d} L_{s}
$$

and for every $0<T<\infty$, we have the two following conditions

$$
\begin{aligned}
& \mathbb{E}\left[\int_{0}^{T} \mathbb{I}_{\left\{X_{s} \in \partial D\right\}} \mathrm{d} s\right]=0 \\
& \mathbb{E}\left[\int_{0}^{T}\left|\nabla \Phi\left(X_{s}\right)\right| \mathrm{d} s\right]<\infty .
\end{aligned}
$$

The previous problem will be called $S V I\left(\Phi ; g ; \sigma ; x_{0}\right)$.

Remark. In the case of the Example 1, we can still define the same kind of problem but without the term $\nabla \Phi\left(X_{t}\right) \mathrm{d} t$ in the SDE (note that $\Phi$ is constant in this case) and without the conditions (2.15) and (2.16): it will be called $R S V I\left(g ; \sigma ; x_{0}\right)$.

\subsection{Results}

We can use the general theorem of existence/uniqueness for multivalued stochastic differential equations obtained in [2] (see also [4] for a complement on the representation of the solutions) to state.

Theorem 2.2. Under the previous assumptions on the data, the problem $S V I\left(\Phi ; g ; \sigma ; x_{0}\right)$ has an unique (strong) solution. Moreover, when $\Phi$ is associated with a real convex function $\phi$ in the sense of (2.3), then inequality (2.16) is strenghtened into

$$
\mathbb{E}\left[\int_{0}^{T}\left|\phi^{\prime}\left(X_{s}^{(k)}-X_{s}^{(j)}\right)\right| \mathrm{d} s\right]<\infty
$$

for every $0<T<\infty$ and $j<k$, and the boundary process is identically zero. 
Proof. Given the general theorem in [2], it remains only to prove the results concerning the situation covered by the setting $(2.3)$. Inequalities $(2.5,2.17)$ are not stated in [2]; inequality $(2.17)$ is obtained with the method used to show (2.16) but here the trick is to use the stronger inequality (2.5) instead of (2.1). Let us first give this proof.

Step 1: Proof of (2.17). Set

$$
S_{p}=\inf \left\{t \geqslant 0:\left|X_{t}\right| \geqslant p\right\} ; \quad p \in \mathbb{N}^{*} .
$$

Then $S_{p}$ is a $\left\{\mathcal{F}_{t}\right\}$ - stopping time for all $p \in \mathbb{N}^{*}$ and $S_{p} \uparrow \infty$ when $p \uparrow \infty$. Applying Itô's formula, we obtain for $a \in D$ and $0 \leqslant s \leqslant t<\infty$ :

$$
\begin{aligned}
\frac{1}{2}\left|X_{s \wedge S_{p}}-a\right|^{2}= & \frac{1}{2}\left|x_{0}-a\right|^{2}+\int_{0}^{s \wedge S_{p}}\left\langle g\left(X_{u}\right), X_{u}-a\right\rangle \mathrm{d} u \\
& +\int_{0}^{s \wedge S_{p}}\left\langle X_{u}-a, \sigma\left(X_{u}\right) \cdot \mathrm{d} B_{u}\right\rangle-\int_{0}^{s \wedge S_{p}}\left\langle\nabla \Phi\left(X_{u}\right), X_{u}-a\right\rangle \mathrm{d} u \\
& -\int_{0}^{s \wedge S_{p}}\left\langle n\left(X_{u}\right), X_{u}-a\right\rangle \mathrm{d} L_{u}+\frac{1}{2} \int_{0}^{s \wedge S_{p}} \operatorname{tr}\left(\sigma \sigma^{*}\left(X_{u}\right)\right) \mathrm{d} u,
\end{aligned}
$$

and using the elementary inequality

$$
x . y \leqslant \frac{1}{2} x^{2}+\frac{1}{2} y^{2}, \quad \forall x, y \in \mathbb{R},
$$

the assumptions on $g, \sigma$, the inequality (2.5) and the fact that $\left\langle n\left(X_{u}\right), X_{u}-a\right\rangle \geqslant 0$ by definition of the outward normal cone:

$$
\begin{aligned}
\frac{1}{2}\left|X_{s \wedge S_{p}}-a\right|^{2} \leqslant & \frac{1}{2}\left|x_{0}-a\right|^{2}+C t+C \int_{0}^{s \wedge S_{p}}\left|X_{u}-a\right|^{2} \mathrm{~d} u \\
& +\int_{0}^{s \wedge S_{p}}\left\langle X_{u}-a, \sigma\left(X_{u}\right) \cdot \mathrm{d} B_{u}\right\rangle+\delta t \\
& -\alpha \sum_{j<k} \int_{0}^{s \wedge S_{p}}\left|\phi^{\prime}\left(X_{u}^{(k)}-X_{u}^{(j)}\right)\right| \mathrm{d} u+\beta \int_{0}^{s \wedge S_{p}}\left|X_{u}-a\right| \mathrm{d} u .
\end{aligned}
$$

Thanks to Davis inequality, the assumption on $\sigma$ and the same elementary inequality as above, the following estimate is valid:

$$
\begin{aligned}
\mathbb{E}\left[\sup _{0 \leqslant s \leqslant t} \int_{0}^{s \wedge S_{p}}\left\langle\sigma\left(X_{u}\right), X_{u}-a\right\rangle \mathrm{d} B_{u}\right] \leqslant & C \mathbb{E}\left[\left(\int_{0}^{t \wedge S_{p}}\left|X_{u}-a\right|^{4} \mathrm{~d} u\right)^{\frac{1}{2}}\right]+C t^{\frac{1}{2}} \\
\leqslant & \frac{1}{4} \mathbb{E}\left[\sup _{0 \leqslant s \leqslant t}\left|X_{s \wedge S_{p}}-a\right|^{2}\right]+C t^{\frac{1}{2}} \\
& +C \mathbb{E} \int_{0}^{t \wedge S_{p}}\left|X_{u}-a\right|^{2} \mathrm{~d} u .
\end{aligned}
$$

We get

$$
\mathbb{E}\left[\sup _{0 \leqslant s \leqslant t}\left|X_{s \wedge S_{p}}-a\right|^{2}\right] \leqslant C(t+1)+C \int_{0}^{t} \mathbb{E}\left(\sup _{0 \leqslant v \leqslant u}\left|X_{v \wedge S_{p}}-a\right|^{2}\right) \mathrm{d} u,
$$


Using Gronwall's lemma, we obtain

$$
\mathbb{E}\left[\sup _{0 \leqslant s \leqslant t}\left|X_{s \wedge S_{p}}-a\right|^{2}\right] \leqslant C(t+1) \mathrm{e}^{C t}
$$

and from the monotone convergence theorem:

$$
\mathbb{E}\left[\sup _{0 \leqslant s \leqslant t}\left|X_{s}-a\right|^{2}\right] \leqslant C
$$

Coming back to the first inequality, we are now in a position to conclude.

Step 2: $\quad L=0$ that is to say the explosive drift is enough to keep the diffusion inside the domain. Let us now prove that the boundary term $L=0$. The proof is written in the case $M<\infty$ : the case $M=\infty$ is just an easy adaptation. Let us introduce some notations: for $1 \leqslant j \leqslant k \leqslant N$, we set

$$
\begin{gathered}
\underline{V}(j ; k)=\left\{x: x^{(0)} \leqslant x^{(1)} \leqslant \cdots \leqslant x^{(j-1)}<x^{(j)}=\cdots=x^{(k)}<x^{(k+1)} \leqslant \cdots \leqslant x^{(N)} \leqslant x^{(N+1)}\right\} \\
\underline{I}(j ; k)=\{j, j+1, \ldots, k\}
\end{gathered}
$$

where $x^{(0)}=x^{(N)}-M, x^{(N+1)}=x^{(1)}+M$, and for $1 \leqslant j<k \leqslant N$

$$
\begin{gathered}
\bar{V}(j ; k)=\left\{x: x^{(1)}=\cdots=x^{(j)}<x^{(j+1)} \leqslant \cdots \leqslant x^{(k-1)}<x^{(k)}=\cdots=x^{(N)}=x^{(1)}+M\right\} \\
\bar{I}(j ; k)=\{k, k+1, \ldots, N, 1,2, \ldots, j\} \\
G_{t}=\int_{0}^{t} n\left(X_{s}\right) \mathrm{d} L_{s}, 0 \leqslant t<\infty
\end{gathered}
$$

whose coordinates are $\left(G^{(1)}, G^{(2)}, \ldots, G^{(N)}\right)$ and we wish to show that $G$ identically vanishes. The following lemma describes the normals to $D$; its proof is adapted from [4] but we give it for the reader's convenience.

Lemma 2.3. Let $x \in \partial D$ such that $x \in \underline{V}(j ; k)$ (respectively $x \in \bar{V}(j ; k)$ ) and let $n=\left(n^{(1)}, n^{(2)}, \ldots, n^{(N)}\right)$ belong to the outward normal cone of $D$ at $x$. Then $\sum_{l \in \underline{I}(j ; k)} n^{(l)}=0$ (respectively $\left.\sum_{l \in \bar{I}(j ; k)} n^{(l)}=0\right)$.

Proof. The proof is given for the case $\underline{V}(j ; k)$. By definition of the outward normal cone of $D$ at $x$, we have:

$$
\langle x-\alpha, n\rangle \geqslant 0, \quad \forall \alpha \in \bar{D}=\left\{y: y^{(1)} \leqslant y^{(2)} \leqslant \cdots \leqslant y^{(N)} \leqslant y^{(1)}+M\right\} .
$$

Let $\varepsilon>0$ such that $x^{(j-1)}<x^{(j)}-\varepsilon<x^{(k)}+\varepsilon<x^{(k+1)}$. The previous inequality for $\alpha=\left(x^{(1)}, x^{(2)}, \cdots, x^{(j-1)}\right.$, $\left.x^{(j)}-\varepsilon, x^{(j+1)}-\varepsilon, \ldots, x^{(k-1)}-\varepsilon, x^{(k)}-\varepsilon, x^{(k+1)}, \ldots, x^{(N)}\right) \in \bar{D}$ gives $\varepsilon\left(n^{(j)}+n^{(j+1)}+\cdots+n^{(k-1)}+n^{(k)}\right) \geqslant 0$, and with $\alpha=\left(x^{(1)}, x^{(2)}, \cdots, x^{(j-1)}, x^{(j)}+\varepsilon, x^{(j+1)}+\varepsilon, \ldots, x^{(k-1)}+\varepsilon, x^{(k)}+\varepsilon, x^{(k+1)}, \ldots, x^{(N)}\right) \in \bar{D}$, we obtain $\varepsilon\left(n^{(j)}+n^{(j+1)}+\cdots+n^{(k-1)}+n^{(k)}\right) \leqslant 0$. Consequently, we have shown that $n^{(j)}+n^{(j+1)}+\cdots+n^{(k-1)}+n^{(k)}=0$. For the other case, it suffices to adapt the choices of $\alpha$. 
Thanks to the lemma (with $j=k$ ), the measure $d G^{(i)}$ is supported for $2 \leqslant i \leqslant N-1$ by

$$
\left\{0 \leqslant u<\infty: X_{u}^{(i)}=X_{u}^{(i+1)}\right\} \cup\left\{0 \leqslant u<\infty: X_{u}^{(i)}=X_{u}^{(i-1)}\right\}
$$

for $i=1$ by

$$
\left\{0 \leqslant u<\infty: X_{u}^{(1)}=X_{u}^{(2)}\right\} \cup\left\{0 \leqslant u<\infty: X_{u}^{(1)}+M=X_{u}^{(N)}\right\}
$$

for $i=N$ by

$$
\left\{0 \leqslant u<\infty: X_{u}^{(N)}=X_{u}^{(N-1)}\right\} \cup\left\{0 \leqslant u<\infty: X_{u}^{(N)}=X_{u}^{(1)}+M\right\}
$$

Therefore, it is enough to prove that for all $j, k$ such that $i \in \underline{I}(j ; k)$ or $i \in \bar{I}(j ; k)$, then

$$
\mathbb{I}_{\left\{X_{u} \in \underline{V}(j ; k)\right\}} \mathrm{d} G_{u}^{(i)}=0,
$$

or in the other case

$$
\mathbb{I}_{\left\{X_{u} \in \bar{V}(j ; k)\right\}} \mathrm{d} G_{u}^{(i)}=0
$$

Let us suppose for a moment that the following equality is known

$$
\mathbb{I}_{\left\{X_{u} \in \underline{V}(j ; k)\right\}} \mathrm{d} G_{u}^{(l)}=\mathbb{I}_{\left\{X_{u} \in \underline{V}(j ; k)\right\}} \mathrm{d} G_{u}^{(m)}
$$

as soon as $l, m \in \underline{I}(j ; k)$, and for the second case

$$
\mathbb{I}_{\left\{X_{u} \in \bar{V}(j ; k)\right\}} \mathrm{d} G_{u}^{(l)}=\mathbb{I}_{\left\{X_{u} \in \bar{V}(j ; k)\right\}} \mathrm{d} G_{u}^{(m)}
$$

as soon as $l, m \in \bar{I}(j ; k)$. Then, the proof is finished. Indeed, considering $v=\sum_{l \in \underline{I}(j ; k)} e_{l}\left(\right.$ or $\left.v=\sum_{l \in \bar{I}(j ; k)} e_{l}\right)$ where $\left(e_{1}, e_{2}, \ldots, e_{N}\right)$ is the canonical basis of $\mathbb{R}^{N}$, the previous lemma asserts that $v$ is orthogonal to every normal vector at a point of $\underline{V}(j ; k)$ (or similarly of $\bar{V}(j ; k)$ ), which implies directly

$$
\begin{aligned}
& \mathbb{I}_{\left\{X_{u} \in \underline{V}(j ; k)\right\}}\left\langle v, \mathrm{~d} G_{u}\right\rangle=0, \\
& \mathbb{I}_{\left\{X_{u} \in \bar{V}(j ; k)\right\}}\left\langle v, \mathrm{~d} G_{u}\right\rangle=0,
\end{aligned}
$$

and from the definition of $v$

$$
\begin{aligned}
& \sum_{l \in \underline{I}(j ; k)} \mathbb{I}_{\left\{X_{u} \in \underline{V}(j ; k)\right\}} \mathrm{d} G_{u}^{(l)}=0 \\
& \sum_{l \in \bar{I}(j ; k)} \mathbb{I}_{\left\{X_{u} \in \bar{V}(j ; k)\right\}} \mathrm{d} G_{u}^{(l)}=0,
\end{aligned}
$$


and this result gives the conclusion. Consequently, the last element to be proved is the equality (2.34) (and (2.35)). Using the occupation times formula, we claim that for $1 \leqslant l<m \leqslant N$

$$
\int_{0}^{M} L_{t}^{a}\left(X^{(m)}-X^{(l)}\right)\left|\phi^{\prime}(a)\right| \mathrm{d} a=\int_{0}^{t}\left|\phi^{\prime}\left(X_{s}^{(m)}-X_{s}^{(l)}\right)\right|\left[\sigma \sigma_{l l}^{*}-2 \sigma \sigma_{l m}^{*}+\sigma \sigma_{m m}^{*}\right]\left(X_{s}\right) \mathrm{d} s,
$$

where $\left(L^{a}(X)\right)_{a}$ is the family of local times for the real continuous semimartingale $X$. From inequality (2.5), the assumption on $\sigma$ and the continuity of $X$, we deduce

$$
\int_{0}^{t}\left|\phi^{\prime}\left(X_{s}^{(m)}-X_{s}^{(l)}\right)\right|\left[\sigma \sigma_{l l}^{*}-2 \sigma \sigma_{l m}^{*}+\sigma \sigma_{m m}^{*}\right]\left(X_{s}\right) \mathrm{d} s<\infty
$$

and consequently

$$
\int_{0}^{M} L_{t}^{a}\left(X^{(m)}-X^{(l)}\right)\left|\phi^{\prime}(a)\right| \mathrm{d} a<\infty
$$

which implies $L_{t}^{0}\left(X^{(m)}-X^{(l)}\right)=0$ since the function $a \rightarrow\left|\phi^{\prime}(a)\right|$ is not integrable at 0 as a consequence of the explosion of $\phi$ at this point. From the identity

$$
X_{u}^{(m)}-X_{u}^{(l)}=\left(X_{u}^{(m)}-X_{u}^{(l)}\right)^{+},
$$

calculating $\left(X_{u}^{(m)}-X_{u}^{(l)}\right)^{+}$with Tanaka's formula and since $L^{0}\left(X^{(m)}-X^{(l)}\right) \equiv 0$, we have

$$
\int_{0}^{t}\left(\mathrm{~d} G_{u}^{(m)}-\mathrm{d} G_{u}^{(l)}\right)=\int_{0}^{t} \mathbb{I}_{\left\{X_{u}^{(m)}>X_{u}^{(l)}\right\}}\left(\mathrm{d} G_{u}^{(m)}-\mathrm{d} G_{u}^{(l)}\right),
$$

where we have used (2.15) to assert that $\int_{0}^{t} \mathbb{I}_{\left\{X_{s}^{(m)}-X_{s}^{(l)}=0\right\}} \mathrm{d} s=0$ in order to identify the others terms in the semimartingale decompositions of $X_{u}^{(m)}-X_{u}^{(l)}$ and $\left(X_{u}^{(m)}-X_{u}^{(l)}\right)^{+}$. The last equality can be written

$$
\int_{0}^{t} \mathbb{I}_{\left\{X_{u}^{(l)}=X_{u}^{(m)}\right\}} \mathrm{d} G_{u}^{(m)}=\int_{0}^{t} \mathbb{I}_{\left\{X_{u}^{(l)}=X_{u}^{(m)}\right\}} \mathrm{d} G_{u}^{(l)},
$$

hence also (2.34). Let us considering now the proof of (2.35). The proof will be identical if $k \leqslant l<m \leqslant N$ or if $1 \leqslant l<m \leqslant j$ and differences only appear when $l \leqslant j$ and $m \geqslant k$. Using the same method as before and the fact that here the function $a \rightarrow\left|\phi^{\prime}(a)\right|$ is not integrable at $M$ (as a consequence of the explosion of $\phi$ at this point), the occupation times formula essentially enables us to obtain $L_{t}^{M^{-}}\left(X^{(m)}-X^{(l)}\right)=0$. Using the general relation $L_{t}^{a}(-X)=L_{t}^{(-a)^{-}}(X)([18]$, p. 217), the following equalities hold:

$$
L_{t}^{M^{-}}\left(X^{(m)}-X^{(l)}\right)=L_{t}^{-M}\left(X^{(l)}-X^{(m)}\right)=L_{t}^{0}\left(X^{(l)}+M-X^{(m)}\right) .
$$

From the identity

$$
X_{u}^{(l)}+M-X_{u}^{(m)}=\left(X_{u}^{(l)}+M-X_{u}^{(m)}\right)^{+},
$$

calculating $\left(X_{u}^{(l)}+M-X_{u}^{(m)}\right)^{+}$with Tanaka's formula and since $L^{0}\left(X^{(l)}+M-X^{(m)}\right) \equiv 0$, we have

$$
\int_{0}^{t}\left(\mathrm{~d} G_{u}^{(m)}-\mathrm{d} G_{u}^{(l)}\right)=\int_{0}^{t} \mathbb{I}_{\left\{X_{u}^{(m)}<X_{u}^{(l)}+M\right\}}\left(\mathrm{d} G_{u}^{(m)}-\mathrm{d} G_{u}^{(l)}\right),
$$


where we have used again (2.15) to assert that $\int_{0}^{t} \mathbb{I}_{\left\{X_{s}^{(m)}-X_{s}^{(l)}=M\right\}} \mathrm{d} s=0$ in order to identify the others terms in the semimartingale decompositions of $X_{u}^{(l)}+M-X_{u}^{(m)}$ and $\left(X_{u}^{(l)}+M-X_{u}^{(m)}\right)^{+}$. The last equality can be written

$$
\int_{0}^{t} \mathbb{I}_{\left\{X_{u}^{(l)}+M=X_{u}^{(m)}\right\}} \mathrm{d} G_{u}^{(m)}=\int_{0}^{t} \mathbb{I}_{\left\{X_{u}^{(l)}+M=X_{u}^{(m)}\right\}} \mathrm{d} G_{u}^{(l)},
$$

hence also (2.35).

Remark. The general theorem in [2] gives also strong existence and uniqueness for the problem $R S V I\left(g ; \sigma ; x_{0}\right)$.

\subsection{Examples}

Let us now apply this theorem to our previous examples:

Example 1. For $\Phi=\Phi_{r}$, the problem $R S V I\left(g ; \sigma ; x_{0}\right)$ is exactly equivalent to the problem of reflecting diffusion with normal reflection in the convex $\bar{D}$, with the coefficients $g, \sigma$ : see $[12,21,24]$. In this case, the convex function only gives a drift term on the boundary:

$$
\mathrm{d} X_{t}=g\left(X_{t}\right) \mathrm{d} t+\sigma\left(X_{t}\right) \mathrm{d} B_{t}-n\left(X_{t}\right) \mathrm{d} L_{t} .
$$

Example 2. For $N=1, \Phi=\phi_{b}, g=0$ and $\sigma=1$, the boundary term is identically zero and $X$ is the Bessel process $B E S(1+2 \gamma)$

$$
\mathrm{d} X_{t}=\mathrm{d} B_{t}+\frac{\gamma}{X_{t}} \mathrm{~d} t
$$

It is well known that 0 is reached if and only if $\gamma<1 / 2$.

In the same way, for $\Phi=\Phi_{b}$, there is no boundary term $L$ and the process $X$ has been constructed and studied in [4]:

$$
\mathrm{d} X_{t}^{(j)}=g\left(X_{t}^{(j)}\right) \mathrm{d} t+\sigma\left(X_{t}^{(j)}\right) \mathrm{d} B_{t}^{(j)}+\gamma \sum_{1 \leqslant k \neq j \leqslant N} \frac{\mathrm{d} t}{X_{t}^{(j)}-X_{t}^{(k)}}, \quad 1 \leqslant j \leqslant N
$$

Comparing the distance between particles with a Bessel process, we can show that any pair of neighbouring particles collide at finite time a.s. (in the case $g=0, \sigma=1$ ) if $\gamma<1 / 2$. Conversely, it has been proved in [5] and [19] that the particles cannot collide if $\gamma \geqslant 1 / 2$.

Example 3. For $N=1, \Phi=\phi_{l}, g=0$ and $\sigma=1$, the boundary term is identically zero and $X$ is the Legendre process with parameter $\gamma$ living on $[0 ; 2 \pi]$ (see [18], p. 331)

$$
\mathrm{d} X_{t}=\mathrm{d} B_{t}+\frac{\gamma}{2} \cot \left(\frac{X_{t}}{2}\right) \mathrm{d} t .
$$

Using the method of the scale function (see [11], p. 339 for example), we obtain that 0 and $2 \pi$ are reached if and only if $\gamma<1 / 2$. Let us notice that the process $J_{t}=\cos \left(\frac{X_{t}}{2}\right)$ is then a Jacobi process on $[-1 ; 1]$ : see [8].

In the same way, for $\Phi=\Phi_{l}$, there is no boundary term $L$ and the process $X$ will be studied in this paper for $g=0$ and $\sigma=I d$ (we could obtain more generality but simple coefficients are necessary in the following to study the system): 
Corollary 2.4. For every $N \in \mathbb{N}$ with $N \geqslant 2, \gamma>0,-\infty<x_{0}^{(1)} \leqslant x_{0}^{(2)} \leqslant \cdots \leqslant x_{0}^{(N)} \leqslant x_{0}^{(1)}+2 \pi$, there is a unique $X=\left(X^{(1)}, X^{(2)}, \ldots, X^{(N)}\right)$ which is the strong solution of the following stochastic differential system:

$$
\begin{cases}\mathrm{d} X_{t}^{(1)} & =\mathrm{d} B_{t}^{(1)}+\frac{\gamma}{2} \sum_{1 \leqslant k \neq 1 \leqslant N} \cot \left(\frac{X_{t}^{(1)}-X_{t}^{(k)}}{2}\right) \mathrm{d} t \\ \cdots & =\cdots \\ \mathrm{d} X_{t}^{(j)} & =\mathrm{d} B_{t}^{(j)}+\frac{\gamma}{2} \sum_{1 \leqslant k \neq j \leqslant N} \cot \left(\frac{X_{t}^{(j)}-X_{t}^{(k)}}{2}\right) \mathrm{d} t \\ \cdots & =\cdots \\ \mathrm{d} X_{t}^{(N)} & =\mathrm{d} B_{t}^{(N)}+\frac{\gamma}{2} \sum_{1 \leqslant k \neq N \leqslant N} \cot \left(\frac{X_{t}^{(N)}-X_{t}^{(k)}}{2}\right) \mathrm{d} t\end{cases}
$$

under the conditions:

$$
\begin{gathered}
X_{0}=\left(x_{0}^{(1)}, x_{0}^{(2)}, \ldots, x_{0}^{(N)}\right) \\
X_{t}^{(1)} \leqslant X_{t}^{(2)} \leqslant \cdots \leqslant X_{t}^{(N)} \leqslant X_{t}^{(1)}+2 \pi, 0 \leqslant t<\infty, \mathbb{P}-\text { a.s. }
\end{gathered}
$$

The issue of collisions of particles in this multidimensional setting will be studied in the next section.

Remark. Using a deterministic time change and the scaling property of the Brownian motion $B$, we may study the system with $\sigma . B$ (for $0<\sigma<\infty$ ) instead of $B$, so it is clear that one parameter $\gamma$ is enough.

Example 4. For $N=1, \Phi=\phi_{h}, g=0$ and $\sigma=1$, the boundary term is identically zero and $X$ is the following process living on $[0 ;+\infty[$

$$
\mathrm{d} X_{t}=\mathrm{d} B_{t}+\gamma \operatorname{coth}\left(X_{t}\right) \mathrm{d} t
$$

Using again the method of the scale function, we obtain that 0 is reached with positive probability if and only if $\gamma<1 / 2$.

In the last section of this paper, we will state the results concerning the situation $\Phi=\Phi_{h}, g=0$ and $\sigma=I d$, which gives the system

$$
\mathrm{d} X_{t}^{(j)}=\mathrm{d} B_{t}^{(j)}+\gamma \sum_{1 \leqslant k \neq j \leqslant N} \operatorname{coth}\left(X_{t}^{(j)}-X_{t}^{(k)}\right) \mathrm{d} t, \quad 1 \leqslant j \leqslant N
$$

The same necessary and sufficient condition for the existence of collisions $(\gamma<1 / 2)$ will be given there.

\section{Collisions}

In the four following sections, we consider the system (2.53). It is quite natural to wonder whether the particles $Z^{(j)}=\mathrm{e}^{i X^{(j)}}$ may collide: the following theorem gives a complete answer:

Theorem 3.1. There are collisions between particles if and only if $\gamma<1 / 2$. More precisely, if $\tau=\inf \{t>$ $0: Z_{t}^{(j)}=Z_{t}^{(k)}$ for $\left.1 \leqslant j \neq k \leqslant N\right\}$, then $\mathbb{P}(\tau<\infty)=1$ or 0 according to $\gamma<1 / 2$ or $\gamma \geqslant 1 / 2$.

Proof. Let us prove the necessary and sufficient conditions separately. Let $\gamma \geqslant 1 / 2$. The proof follows partly [19] and in fact the method has something to do with the ideas used to attack the problem of recurrence for Bessel 
processes as it can be seen in [11] (p. 158). Let us first notice that

$$
\frac{1}{2} \Delta \Phi_{l}(x)-\left|\nabla \Phi_{l}(x)\right|^{2}=\frac{\gamma}{2}\left(\frac{1}{2}-\gamma\right) \sum_{j \neq k} \sin ^{-2}\left(\frac{x_{j}-x_{k}}{2}\right)+C-\frac{\gamma^{2}}{2} \sum_{j, k, l \neq} \cot \left(\frac{x_{j}-x_{k}}{2}\right) \cot \left(\frac{x_{j}-x_{l}}{2}\right)
$$

and, on the other hand, the following circular formula will be useful

$$
\cot (a) \cot (b)+\cot (a) \cot (c)+\cot (b) \cot (c)=1,
$$

as soon as $a+b+c=0$. (An analogous circular formula was used in the case of the interaction $1 / x$ where the right-hand side is 0 and another one is valid for $\operatorname{coth}(x)$ where the right-hand side is -1 .) By convention, if $x \in \mathbb{R}^{N}, x^{(N+1)}=x^{(1)}+2 \pi$. For $0<\varepsilon<\min _{i \neq j}\left|x_{0}^{(j)}-x_{0}^{(i)}\right|$ (with $1 \leqslant i, j \leqslant N+1$ ), one defines

$$
\tau_{\varepsilon}=\inf \left\{t>0:\left|X_{t}^{(i)}-X_{t}^{(j)}\right|=\varepsilon \quad \text { for } \quad 1 \leqslant i \neq j \leqslant N+1\right\}
$$

in the manner that

$$
\tau_{\varepsilon} \uparrow \tau, \quad \text { when } \varepsilon \downarrow 0
$$

Since $\phi_{l}$ is $C^{2}$ in $[\varepsilon ; M-\varepsilon]$, it is possible to apply Itô 's formula and obtain

$$
\Phi_{l}\left(X_{t \wedge \tau_{\varepsilon}}\right)=\Phi_{l}\left(x_{0}\right)+\int_{0}^{t \wedge \tau_{\varepsilon}}\left\langle\nabla \Phi_{l}\left(X_{s}\right), \mathrm{d} B_{s}\right\rangle-\int_{0}^{t \wedge \tau_{\varepsilon}}\left|\nabla \Phi_{l}\left(X_{s}\right)\right|^{2} \mathrm{~d} s+1 / 2 \int_{0}^{t \wedge \tau_{\varepsilon}} \Delta \Phi_{l}\left(X_{s}\right) \mathrm{d} s,
$$

and thanks to $(3.1,3.2)$, it is clear that there exists $C>0$ such that the process $\Phi_{l}\left(X_{t \wedge \tau_{\varepsilon}}\right)-C\left(1+t \wedge \tau_{\varepsilon}\right)$ is a supermartingale, hence particularly

$$
\mathbb{E}\left(\Phi_{l}\left(X_{t \wedge \tau_{\varepsilon}}\right)\right) \leqslant C(1+t)
$$

and considering the event $\mathcal{B}=\left\{\tau_{\varepsilon} \leqslant t\right\}$, the positivity of $\phi_{l}$ and $\Phi_{l}$ give

$$
-\gamma \ln \left(\sin \left(\frac{\varepsilon}{2}\right)\right) \mathbb{P}(\mathcal{B}) \leqslant C
$$

which implies then

$$
\mathbb{P}\left(\tau_{\varepsilon} \leqslant t\right) \rightarrow 0, \quad \text { when } \varepsilon \rightarrow 0
$$

that is to say

$$
\mathbb{P}(\tau \leqslant t)=0
$$

It is now easy to conclude by letting $t$ tend to infinity.

Let $\gamma<1 / 2$. Considering the difference $X_{t}^{(j+1)}-X_{t}^{(j)}$ and having in mind the fact that cot is decreasing on $[0 ; \pi]$ and odd on $[-\pi ; \pi]$, we succeed in finding the sign of the various bounded variation elements of this semimartingale. Let $Y$ the $[0 ; 2 \pi]$-valued solution to

$$
\mathrm{d} Y_{t}=\mathrm{d} B_{t}^{(j+1)}-\mathrm{d} B_{t}^{(j)}+\gamma \cot \left(\frac{Y_{t}}{2}\right) \mathrm{d} t
$$


with $Y_{0}=x_{0}^{(j+1)}-x_{0}^{(j)}$. Using a time scale, it is easy to see that this process has the same boundary properties as the Legendre process in (2.52). Since the process $H_{t}=\left(X_{t}^{(j+1)}-X_{t}^{(j)}\right)-Y_{t}$ has bounded variation, it satisfies: $L_{t}^{0}(H)=0$ and Tanaka's formula takes on the simple form

$$
\begin{aligned}
H_{t}^{+} & =\int_{0}^{t} \mathbb{I}_{\left\{H_{s}>0\right\}}\left[\sum_{k \neq j+1} \frac{\gamma}{2} \cot \left(\frac{X_{s}^{(j+1)}-X_{s}^{(k)}}{2}\right)-\sum_{k \neq j} \frac{\gamma}{2} \cot \left(\frac{X_{s}^{(j)}-X_{s}^{(k)}}{2}\right)-\gamma \cot \left(\frac{Y_{s}}{2}\right)\right] \mathrm{d} s \\
& \leqslant \gamma \int_{0}^{t} \mathbb{I}_{\left\{H_{s}>0\right\}}\left[\cot \left(\frac{X_{s}^{(j+1)}-X_{s}^{(j)}}{2}\right)-\cot \left(\frac{Y_{s}}{2}\right)\right] \mathrm{d} s \leqslant 0 .
\end{aligned}
$$

This proves

$$
0 \leqslant X_{t}^{(j+1)}-X_{t}^{(j)} \leqslant Y_{t}
$$

and an analogous proof yields

$$
Y_{t} \leqslant X_{t}^{(N)}-X_{t}^{(1)} \leqslant 2 \pi .
$$

As 0 and $2 \pi$ are reached a.s. by $Y$ if $\gamma<1 / 2$, we deduce the existence of collisions for particles in this case.

\section{Convergence}

We will now consider the behaviour of the interacting particles system (2.53) when

$$
\gamma=\frac{2 \lambda}{N}
$$

(for $\lambda>0$ ) as the number $N$ of particles tends to infinity, which we study through the empirical measure process:

$$
\mu_{t}^{(N)}=\frac{1}{N} \sum_{j=1}^{N} \delta_{Z_{t}^{(j)}}
$$

We consider each $\mu_{t}^{(N)}$ as a probability measure on $[-\pi ; \pi[$ after homeomorphic identification of $[-\pi ; \pi[$ with the unit circle. Henceforth, we will consider initial conditions for the particles still given by (2.54) such that

$$
\mu_{0}^{(N)} \underset{N \rightarrow \infty}{\longrightarrow} \mu_{0}
$$

where $\mu_{0}$ is a probability measure on $\left[-\pi ; \pi\left[\right.\right.$. The following result gives the weak convergence of $\left(\mu^{(N)}\right)_{N}$ when $N \uparrow \infty$ to a measure - valued process $\mu$.

Theorem 4.1. With the same assumptions as in Corollary 2.4 except $\lambda$ is given by (4.1) and the initial data by (4.3), the sequence of measure-valued processes $\left(\mu^{(N)}\right)_{N}$ is (weakly) convergent and the limit $\mu$ is the unique continuous probability measure-valued function satisfying

$$
\begin{aligned}
\int_{-\pi}^{\pi} f(x) \mu_{t}(\mathrm{~d} x)= & \int_{-\pi}^{\pi} f(x) \mu_{0}(\mathrm{~d} x)+\frac{1}{2} \int_{0}^{t} \mathrm{~d} s\left(\int_{-\pi}^{\pi} f^{\prime \prime}(x) \mu_{s}(\mathrm{~d} x)\right) \\
& +\frac{\lambda}{2} \int_{0}^{t} \mathrm{~d} s\left(\int_{-\pi}^{\pi} \int_{-\pi}^{\pi}\left(f^{\prime}(x)-f^{\prime}(y)\right) \cot \left(\frac{x-y}{2}\right) \mu_{s}(\mathrm{~d} x) \mu_{s}(\mathrm{~d} y)\right)
\end{aligned}
$$

for all $f \in C^{2}(\mathbb{R}) 2 \pi$-periodic. 
Proof. First part: tightness.

In order to obtain the tightness of $\left(\mu^{(N)}\right)_{N}$, it is sufficient as in [5] to prove that the sequence of continuous real-valued processes $\left(\int f(x) \mathrm{d} \mu^{(N)}(x)\right)_{N}$ is tight for all $f \in C^{2}(\mathbb{R})$ being $2 \pi$-periodic. From $(2.53)$ and Itô's formula, we have:

$$
\begin{aligned}
\int_{-\pi}^{\pi} f(x) \mu_{t}^{(N)}(\mathrm{d} x)= & \frac{1}{N} \sum_{j=1}^{N} f\left(X_{t}^{(j)}\right)=\frac{1}{N} \sum_{j=1}^{N} f\left(x_{0}^{(j)}\right)+\frac{1}{N} \sum_{j=1}^{N} \int_{0}^{t} f^{\prime}\left(X_{s}^{(j)}\right) \mathrm{d} B_{s}^{(j)} \\
& +\frac{1}{N} \sum_{j=1}^{N} \int_{0}^{t} f^{\prime}\left(X_{s}^{(j)}\right)\left(\frac{\lambda}{N} \sum_{1 \leqslant k \neq j \leqslant N} \cot \left(\frac{X_{s}^{(j)}-X_{s}^{(k)}}{2}\right)\right) \mathrm{d} s \\
& +\frac{1}{2 N} \sum_{j=1}^{N} \int_{0}^{t} f^{\prime \prime}\left(X_{s}^{(j)}\right) \mathrm{d} s .
\end{aligned}
$$

Hence, using the definition $(4.2)$ of $\left(\mu^{(N)}\right)_{N}$ and the symmetry of the interaction, the above equality may also be written:

$$
\begin{aligned}
\int_{-\pi}^{\pi} f(x) \mu_{t}^{(N)}(\mathrm{d} x)= & \int_{-\pi}^{\pi} f(x) \mu_{0}^{(N)}(\mathrm{d} x)+M_{t}^{(N)} \\
& +\frac{\lambda}{2} \int_{0}^{t} \mathrm{~d} s\left(\iint_{\{x \neq y\}}\left(f^{\prime}(x)-f^{\prime}(y)\right) \cot \left(\frac{x-y}{2}\right) \mu_{s}^{(N)}(\mathrm{d} x) \mu_{s}^{(N)}(\mathrm{d} y)\right) \\
& +\frac{1}{2} \int_{0}^{t} \mathrm{~d} s\left(\int_{-\pi}^{\pi} f^{\prime \prime}(x) \mu_{s}^{(N)}(\mathrm{d} x)\right)
\end{aligned}
$$

where $M^{(N)}$ is a continuous martingale such that:

$$
\left\langle M^{(N)}\right\rangle_{t}=\frac{1}{N^{2}} \sum_{j=1}^{N} \int_{0}^{t}\left\{f^{\prime}\left(X_{s}^{(j)}\right)\right\}^{2} \mathrm{~d} s
$$

Taking into account the fact that $\left(f^{\prime}(x)-f^{\prime}(y)\right) \cot \left(\frac{x-y}{2}\right) \rightarrow 2 f^{\prime \prime}(z)$ when $(x, y) \rightarrow(z, z)$, the triple integral in $(4.6)$ is

$$
\frac{\lambda}{2} \int_{0}^{t} \mathrm{~d} s\left(\int_{-\pi}^{\pi} \int_{-\pi}^{\pi}\left(f^{\prime}(x)-f^{\prime}(y)\right) \cot \left(\frac{x-y}{2}\right) \mu_{s}^{(N)}(\mathrm{d} x) \mu_{s}^{(N)}(\mathrm{d} y)\right)-\frac{\lambda}{N} \int_{0}^{t} \mathrm{~d} s\left(\int_{-\pi}^{\pi} f^{\prime \prime}(x) \mu_{s}^{(N)}(\mathrm{d} x)\right)
$$

so that

$$
\begin{aligned}
\int_{-\pi}^{\pi} f(x) \mu_{t}^{(N)}(\mathrm{d} x)= & \int_{-\pi}^{\pi} f(x) \mu_{0}^{(N)}(\mathrm{d} x)+M_{t}^{(N)} \\
& +\frac{\lambda}{2} \int_{0}^{t} \mathrm{~d} s\left(\int_{-\pi}^{\pi} \int_{-\pi}^{\pi}\left(f^{\prime}(x)-f^{\prime}(y)\right) \cot \left(\frac{x-y}{2}\right) \mu_{s}^{(N)}(\mathrm{d} x) \mu_{s}^{(N)}(\mathrm{d} y)\right) \\
& -\frac{\lambda}{N} \int_{0}^{t} \mathrm{~d} s\left(\int_{-\pi}^{\pi} f^{\prime \prime}(x) \mu_{s}^{(N)}(\mathrm{d} x)\right) \\
& +\frac{1}{2} \int_{0}^{t} \mathrm{~d} s\left(\int_{-\pi}^{\pi} f^{\prime \prime}(x) \mu_{s}^{(N)}(\mathrm{d} x)\right)
\end{aligned}
$$


Now, using (4.9), the assumption on $f$ and Aldous criterion applied to the four processes in the righthand side, the sequence of continuous real-valued processes $\left(\int f(x) \mu^{(N)}(\mathrm{d} x)\right)_{N}$ is easily shown to be tight and, consequently, the laws of the processes $\left.\left(\mu^{(N)}\right)\right)_{N}$ are tight. From the tightness, we have at least convergence $\mu^{(N)} \Rightarrow \mu$ along a subsequence $\left(N_{k}\right)$. Let $k$ tend to infinity in (4.9) (written for $N=N_{k}$ ) for suitable $f$ and use the convergence $\mu^{\left(N_{k}\right)} \Rightarrow \mu$ : thus, we show that any limit process $\mu$ satisfies (4.4).

Second part: convergence.

From the first part, it remains to prove there is only one possible limit for all subsequences $\left(\mu^{\left(N_{k}\right)}\right)_{k}$. More precisely, we know that $\mu^{(N)} \Rightarrow \mu$ along a subsequence and any such limit process $\mu=\left\{\mu_{t}, t \in \mathbb{R}^{+}\right\}$satisfies (4.4). If we can show that (4.4) has a unique solution, we actually prove the convergence of $\left(\mu^{(N)}\right)_{N}$, this time not only up to subsequences, and thereby Theorem 4.1. To achieve this, we will prove that the Fourier coefficients of a solution $\mu$ to (4.4) are uniquely determined: it is the reason why we apply (4.4) with the test function $x \rightarrow e^{-i n x}$ so that

$$
\left\langle\mu_{t}, f\right\rangle=\int f(x) \mathrm{d} \mu_{t}(x)=\hat{\mu}_{t}(n)
$$

Using the basic formula

$$
\cot \left(\frac{x-y}{2}\right)=i \frac{\mathrm{e}^{i x}+\mathrm{e}^{i y}}{\mathrm{e}^{i x}-\mathrm{e}^{i y}}
$$

elementary calculus shows that

$$
\frac{\mathrm{d}}{\mathrm{d} t} \hat{\mu}_{t}(n)=-n(\lambda+n / 2) \hat{\mu}_{t}(n)-\lambda n \sum_{k=1}^{n-1} \hat{\mu}_{t}(k) \hat{\mu}_{t}(n-k)
$$

and, by induction, it is now easy to get uniqueness for (4.12) and so for (4.4). This ends the proof of Theorem 4.1.

Remark. It is clear that the tightness result (and the convergence in the next section) is obtained for $\mu^{(N)}$ being random variables with values in $C\left(\mathbb{R}^{+} ; M_{1}(\mathbb{R})\right)\left(M_{1}(E)\right.$ : probability measures on $\left.E\right)$, that is to say at the level of the time-marginals, and not at the level of the processes when one works with $M_{1}\left(C\left(\mathbb{R}^{+} ; \mathbb{R}\right)\right)$ as a state space. The problem of propagation of chaos for this class of singular model is not treated in this paper.

Remark. The method used here is very similar to the proof in [5] in an analogous situation: the test function $x \rightarrow x^{n}$ (giving moments) is naturally replaced by $x \rightarrow \mathrm{e}^{-i n x}$ (giving Fourier coefficients).

\section{Limit Distribution}

It is quite tempting to study the convergence of $\mu_{t}$ when $t \uparrow \infty \ldots$ and to guess that the uniform distribution will be the only possible limit. Indeed, this conjecture will be shown to be true. Before its proof, let us recall that for the Brownian particles with electrostatic repulsion in $\mathbb{R}[5]$ and [19] solve the similar problem of limit distribution (with a repelling force in the drift of (1.1) to prevent the particules from escaping to infinity), showing that the semicircular Wigner law is a limit ditribution and is the only one with all finite moments. Their results remind us of the link between Brownian particles with electrostatic repulsion, eigenvalues of random matrices with Brownian motion entries on the one hand and, on the other hand, the key role of the Wigner law in the theory of random matrices theory as stated in the classical Wigner's theorem and Voiculescu's theorem in the free probability theory: see [14] and [25]. 
Theorem 5.1. When $t \uparrow \infty$, the probability $\mu_{t}$ obtained in Theorem 4.1 converges towards the uniform probability on $[-\pi ; \pi[$.

Proof. Existence of a limit distribution is obvious since the state space here is compact. Let us now show uniqueness and, in the same way, the nature of this law. The differential equations (4.12) allows us to get the explicit expression of $\hat{\mu}_{t}(n)$ as a sum of exponentials terms which vanish as $t \uparrow \infty$, therefore the only non-zero Fourier coefficient is $\hat{\mu}_{t}(0)=1$ and the uniform distribution is the only limit.

\section{Existence of A SMooth Density}

It was shown in [1] in the case of real electrostatic Brownian particles that the probability $\mu_{t}$ has a smooth density. More precisely, starting from (4.4) with $x_{0}=0$, it was known that $\mu$ is the only weak solution of the following limiting non-linear second-order integro-partial differential equation

$$
\left\{\begin{array}{l}
\frac{\partial \mu_{t}}{\partial t}=\frac{1}{2} \frac{\partial^{2} \mu_{t}}{\partial x^{2}}-2 \lambda \frac{\partial\left(\mu_{t} \mathcal{H}\left(\mu_{t}\right)\right)}{\partial x} \\
\mu_{0}=\delta_{0}
\end{array}\right.
$$

the so-called McKean-Vlasov equation, where $\mathcal{H}$ stands for the Hilbert transform of $\mu_{t}$ given by $\mathcal{H}(\nu)=$ $p v\left(\frac{1}{x}\right) * \nu$. We proved, using complex variable methods, that, for each $t>0$, the probability $\mu_{t}$ has a density $u(t,)=.u_{t}$, and that $u_{t}$ and its Hilbert transform $\mathcal{H}\left(u_{t}\right)$ are real analytic. Thus, $u=\left(u_{t}\right)_{t}$ is a classical solution of

$$
\left\{\begin{array}{l}
\frac{\partial u}{\partial t}=\frac{1}{2} \frac{\partial^{2} u}{\partial x^{2}}-2 \lambda \frac{\partial(u \mathcal{H}(u))}{\partial x} \\
u(t, x) \mathrm{d} x \rightarrow \delta_{0} \text { as } t \rightarrow 0
\end{array}\right.
$$

Theorem 6.1. Let $\mu_{0}$ be a probability-measure on $\mathbb{R}$. There is exactly one (classical) solution $u$ for

$$
\left\{\begin{array}{l}
\frac{\partial u_{t}}{\partial t}=\frac{1}{2} \frac{\partial^{2} u}{\partial x^{2}}-2 \lambda \frac{\partial(u \mathbb{H}(u))}{\partial x} \\
u(t, x) \mathrm{d} x \rightarrow \mu_{0} \text { as } t \rightarrow 0
\end{array}\right.
$$

The function $u_{t}$ is the density of the probability measure $\mu_{t}$ introduced in the Theorem 4.1. Moreover, $u$ and its Hilbert transform $\mathbb{H}(u)$ are real analytic functions in $(t, x) \in \mathbb{R}_{+}^{*} \times\left[-\pi ; \pi\left[\right.\right.$. For the particular case $\mu_{0}=\delta_{0}$, we have:

$$
\mathbb{H}(u(t, .))(x)=\frac{1}{2 \lambda t} \lim _{y \rightarrow 0} \mathcal{R} e \frac{\int(x-v) \mathrm{e}^{-\frac{(x-v)^{2}}{2 t}} \frac{\mathrm{d} v}{R(v, y)}}{\int \mathrm{e}^{-\frac{(x-v)^{2}}{2 t}} \frac{\mathrm{d} v}{R(v, y)}}
$$

where $R(v, y)=\sum_{k=-\infty}^{k=+\infty}\left[\sin \left(\frac{v-2 k \pi+i y}{2}\right)\right]^{2 \lambda} \mathrm{e}^{2 i k \pi \lambda} \mathbb{I}_{\{(2 k-1) \pi \leqslant v<(2 k+1) \pi\}}$, with the principal branch for the power function.

Remark. There is a similar way to write $u(t, x)$. The proof below uses the method initiated in [1] but let us notice that the present situation makes clear the periodic properties, the key role of the Poisson kernel in the disk and some typical features in the estimates (uniqueness for the heat equation in a good class of solutions, validity of Cauchy's formula). 
Proof. For $z=x+i y \in \mathbb{C}, y>0$, we consider the function

$$
M_{t}(z)=\frac{1}{2} \int_{-\pi}^{\pi} \cot \left(\frac{z-u}{2}\right) \mu_{t}(\mathrm{~d} u)
$$

which is holomorphic in the upper half-plane. Simple calculations, with (4.4) written for the function $f(u)=$ $\frac{1}{2} \cot \left(\frac{z-u}{2}\right)$, show that $M$ satisfies the holomorphic Burgers equation

$$
\left\{\begin{array}{l}
\frac{\partial M_{t}(z)}{\partial t}=\frac{1}{2} M_{t}^{\prime \prime}(z)-2 \lambda M_{t}(z) \cdot M_{t}^{\prime}(z) \\
M_{0}(z)=\frac{1}{2} \int_{-\pi}^{\pi} \cot \left(\frac{z-u}{2}\right) \mu_{0}(\mathrm{~d} u)
\end{array}\right.
$$

where $F^{\prime}(z)$ denotes for a holomorphic function $F$ its derivative with respect to the complex variable $z$. Using the Poisson kernel in the disk, it is easy to show that $M_{t}(.+i y)$ converges in the sense of distributions in the $x \in[-\pi ; \pi[$ variable to

$$
\frac{1}{2} p v\left(\cot \left(\frac{x}{2}\right)\right) * \mu_{t}-i \pi \mu_{t}
$$

as $y$ tends to 0 . Here $p v\left(\cot \left(\frac{x}{2}\right)\right)$ is the Schwartz distribution $f \longrightarrow \lim _{\varepsilon \rightarrow 0} \int_{\varepsilon<|x|<\pi} f(x) \cot \left(\frac{x}{2}\right) \mathrm{d} x$, and by the very definition of the periodic Hilbert transform $\mathbb{H}$,

$$
\mathbb{H}\left(\mu_{t}\right)=\frac{1}{2} p v\left(\cot \left(\frac{x}{2}\right)\right) * \mu_{t}
$$

We set $N_{t}(z)=\int_{i}^{z} M_{t}(v) \mathrm{d} v$, which defines a holomorphic function in the upper half-plane such that $N_{t}^{\prime}(z)=$ $M_{t}(z)$ and $N_{t}(z+2 \pi)=N_{t}(z)-i \pi$. Moreover, after some calculations, we obtain

$$
\mathcal{R} e N_{t}(z) \geqslant \ln (\sinh (y / 2))-\ln (\cosh (1 / 2)) .
$$

Following the Hopf-Cole transformation, we define the new function

$$
H_{t}(z)=\exp \left(-2 \lambda N_{t}(z)\right)
$$

which is holomorphic in the upper half-plane and satisfies

$$
H_{t}^{\prime}(z)=-2 \lambda M_{t}(z) H_{t}(z) .
$$

We then obtain

$$
\left(\frac{\partial N_{t}}{\partial t}-\frac{1}{2} N_{t}^{\prime \prime}+\lambda M_{t}^{2}\right)^{\prime}=0
$$

so that

$$
\frac{\partial N_{t}}{\partial t}-\frac{1}{2} N_{t}^{\prime \prime}+\lambda M_{t}^{2}=f(t)
$$


for a given continuous function $f: \mathbb{R}_{+} \rightarrow \mathbb{C}$. We now set $K_{t}=g(t) H_{t}$, where the function $g: \mathbb{R}_{+} \rightarrow \mathbb{C}$ is such that $\dot{g}=2 \lambda f g$ and $g(0)=1$ (the function $g$ does not vanish). Thus $K$ is the unique bounded solution (in $x$, for fixed $y$ ) to

$$
\frac{\partial K_{t}}{\partial t}(z)-\frac{1}{2} \frac{\partial^{2} K_{t}}{\partial x^{2}}(z)=0
$$

Therefore, if we set $G_{t}(z)=\frac{1}{\sqrt{2 \pi t}} \exp \left(-\frac{z^{2}}{2 t}\right), K_{t}$ is given by the formula

$$
K_{t}(x+i y)=\int_{\mathbb{R}} H_{0}(u+i y) G_{t}(x-u) \mathrm{d} u,
$$

which becomes, using Cauchy Formula

$$
\begin{aligned}
K_{t}(x+i y) & =\int_{\mathbb{R}} H_{0}(u+i y) G_{t}(x-u) \mathrm{d} u=\int_{z=u+i y} H_{0}(z) G_{t}(x+i y-z) \mathrm{d} z \\
& =\int_{z=u+i} H_{0}(z) G_{t}(x+i y-z) \mathrm{d} z=\int_{\mathbb{R}} H_{0}(u+i) G_{t}(x+i y-i-u) \mathrm{d} u
\end{aligned}
$$

so that $K_{t}$ is the restriction to the upper half-plane of the holomorphic function (also denoted by $K$ ) defined over the whole complex plane by

$$
K_{t}(z)=\int_{\mathbb{R}} H_{0}(u+i) G_{t}(z-i-u) \mathrm{d} u .
$$

We already know that $K_{t}$ does not vanish in the upper half-plane. Let us suppose that $K_{t}\left(x_{0}\right)=0$ for $x_{0}$ on the real axis. Since $K_{t}$ is holomorphic, there exist $k \in \mathbb{N}^{*}$ and a holomorphic function $R$ such that $R$ does not vanish in the ball $B\left(x_{0} ; \delta\right)$ and

$$
K_{t}(z)=\left(z-x_{0}\right)^{k} R(z)
$$

Thus, for $z \in B\left(x_{0} ; \delta\right) \backslash\left\{x_{0}\right\}$,

$$
\frac{K_{t}^{\prime}(z)}{K_{t}(z)}=\frac{k}{z-x_{0}}+\frac{R^{\prime}(z)}{R(z)}
$$

In particular, for $0<\varepsilon<\delta$

$$
\frac{K_{t}^{\prime}\left(x_{0}+i \varepsilon\right)}{K_{t}\left(x_{0}+i \varepsilon\right)}=-\frac{k}{\varepsilon} i+O(1),
$$

when $\varepsilon$ tends to 0 . On the other hand, we have for $y>0$

$$
\frac{K_{t}^{\prime}(z)}{K_{t}(z)}=-2 \lambda M_{t}(z) .
$$

But the imaginary part of $M_{t}(z)$ is negative since it is equal to

$$
-\frac{1}{2}\left(1-\mathrm{e}^{-2 y}\right) \int_{-\pi}^{\pi} \frac{\mu_{t}(\mathrm{~d} u)}{1+\mathrm{e}^{-2 y}-2 \mathrm{e}^{-y} \cos (x-u)} .
$$


We get a contradiction. Finally, $K_{t}$ never vanishes on $\mathbb{R}$ and we get on $[-\pi ; \pi[$

$$
\begin{aligned}
\mu_{t}(\mathrm{~d} x) & =\frac{1}{2 \lambda \pi} \mathcal{I} m\left(\frac{K_{t}^{\prime}(x)}{K_{t}(x)}\right) \mathrm{d} x, \\
\mathbb{H}\left(\mu_{t}\right) & =-\frac{1}{2 \lambda} \mathcal{R} e\left(\frac{K_{t}^{\prime}(x)}{K_{t}(x)}\right) .
\end{aligned}
$$

Since $\frac{K_{t}^{\prime}(z)}{K_{t}(z)}$ is analytic in a neighborhood of the closed upper half-plane, both $\mu_{t}$ and its Hilbert transform $\mathbb{H}\left(\mu_{t}\right)$ possess a real analytic density. This ends the proof of theorem.

\section{THE HYPERBOLIC CASE}

Using the above remarks, we can state the following result for the situation when the interaction cot is replaced by coth. Since the proof is quite similar (see also the case $1 / x$ in $[1,4]$ ), we only give the typical features corresponding to this interaction.

Theorem 7.1. For every $N \in \mathbb{N}$ with $N \geqslant 2, \gamma>0,-\infty<x_{0}^{(1)} \leqslant x_{0}^{(2)} \leqslant \cdots \leqslant x_{0}^{(N)}<\infty$, there is a unique $X=\left(X^{(1)}, X^{(2)}, \ldots, X^{(N)}\right)$ which is the strong solution of the following stochastic differential system:

$$
\begin{cases}\mathrm{d} X_{t}^{(1)} & =\mathrm{d} B_{t}^{(1)}+\gamma \sum_{1 \leqslant k \neq 1 \leqslant N} \operatorname{coth}\left(X_{t}^{(1)}-X_{t}^{(k)}\right) \mathrm{d} t \\ \cdots & =\cdots \\ \mathrm{d} X_{t}^{(j)} & =\mathrm{d} B_{t}^{(j)}+\gamma \sum_{1 \leqslant k \neq j \leqslant N} \operatorname{coth}\left(X_{t}^{(j)}-X_{t}^{(k)}\right) \mathrm{d} t \\ \cdots & =\cdots \\ \mathrm{d} X_{t}^{(N)} & =\mathrm{d} B_{t}^{(N)}+\gamma \sum_{1 \leqslant k \neq N \leqslant N} \operatorname{coth}\left(X_{t}^{(N)}-X_{t}^{(k)}\right) \mathrm{d} t\end{cases}
$$

under the conditions:

$$
\begin{gathered}
X_{0}=\left(x_{0}^{(1)}, x_{0}^{(2)}, \ldots, x_{0}^{(N)}\right) \\
X_{t}^{(1)} \leqslant X_{t}^{(2)} \leqslant \cdots \leqslant X_{t}^{(N)}, 0 \leqslant t<\infty, \mathbb{P}-\text { a.s. }
\end{gathered}
$$

There are collisions with positive probability between particules if and only if $\gamma<1 / 2$.

Assume now (4.1-4.3). The sequence of measure-valued process $\left(\mu^{(N)}\right)_{N}$ is (weakly) convergent and the limit $\mu$ is the unique continuous probability measure-valued function satisfying:

$$
\begin{aligned}
\int f(x) \mu_{t}(\mathrm{~d} x)= & \int f(x) \mu_{0}(\mathrm{~d} x)+\frac{1}{2} \int_{0}^{t} \mathrm{~d} s\left(\int f^{\prime \prime}(x) \mu_{s}(\mathrm{~d} x)\right) \\
& +\lambda \int_{0}^{t} \mathrm{~d} s\left(\iint\left(f^{\prime}(x)-f^{\prime}(y)\right) \operatorname{coth}(x-y) \mu_{s}(\mathrm{~d} x) \mu_{s}(\mathrm{~d} y)\right)
\end{aligned}
$$

for all $f \in C_{b}^{2}(\mathbb{R})$. 
For a probability-measure $\nu$ on $\mathbb{R}$, we define the hyperbolic Hilbert transform $H(\nu)=p v(\operatorname{coth}(x)) * \nu$. Let $\mu_{0}$ a probability-measure on $\mathbb{R}$. Then, there is exactly one (classical) solution u for

$$
\left\{\begin{array}{l}
\frac{\partial u_{t}}{\partial t}=\frac{1}{2} \frac{\partial^{2} u}{\partial x^{2}}-2 \lambda \frac{\partial(u H(u))}{\partial x} \\
u(t, x) \mathrm{d} x \rightarrow \mu_{0} \text { as } t \rightarrow 0
\end{array}\right.
$$

Moreover, $u$ and its Hilbert transform $H(u)$ are real analytic functions in $(t, x) \in \mathbb{R}_{+}^{*} \times \mathbb{R}$. More precisely, if $\mu_{0}=\delta_{0}$, then

$$
H(u(t, .))(x)=\frac{1}{2 \lambda t} \lim _{y \rightarrow 0} \mathcal{R} e \frac{\int(x-v) \mathrm{e}^{-\frac{(x-v)^{2}}{2 t}} \frac{\mathrm{d} v}{[\sinh (v+i y)]^{2 \lambda}}}{\int \mathrm{e}^{-\frac{(x-v)^{2}}{2 t}} \frac{\mathrm{d} v}{[\sinh (v+i y)]^{2 \lambda}}},
$$

with the principal branch for the power function.

Sketch of the proof. The proof goes the same way as the previous ones as we already quoted. Notice however the non vanishing behaviour of $\operatorname{coth}(x)$ as $x$ goes to infinity, leading to a long range interaction between particles. As a consequence, when $\gamma<1 / 2$, there are collisions with positive probability instead of probability one. To prove the smoothness of the solution we set

$$
M_{t}(z)=\int \operatorname{coth}(z-u) \mu_{t}(\mathrm{~d} u)
$$

for $z=x+i y \in \mathbb{C}, 0<y<\pi$. We then have

$$
M_{t}(z)=\int \frac{\mathrm{e}^{4(x-u)}-1-2 i \sin (2 y) \mathrm{e}^{2(x-u)}}{\mathrm{e}^{4(x-u)}+1-2 \cos (2 y) \mathrm{e}^{2(x-u)}} \mu_{t}(\mathrm{~d} u) .
$$

We set $N_{t}(z)=\int_{i \pi / 2}^{z} M_{t}(v) \mathrm{d} v$, which defines a holomorphic function in the strip $\left.\mathbb{R} \times\right] 0 ; \pi\left[\right.$ such that $N_{t}^{\prime}(z)=$ $M_{t}(z)$. Simple calculations prove that $\mathcal{R} e N_{t}(z) \geqslant-|x|+\ln (\sin (y))$. Introducing as usually the holomorphic function $H_{t}(z)=\exp \left(-2 \lambda N_{t}(z)\right)$, we see that the associated function $K_{t}(z)$ is still given by the heat kernel and therefore extends to a holomorphic function on the whole plane. As $y$ goes to zero, the imaginary part of $M_{t}(z)$ is negative, so that the usual argument proves that $K_{t}$ does not vanish on the real line.

We thank Aline Bonami for stimulating discussions on the contents of this paper.

\section{REFERENCES}

[1] A. Bonami, F. Bouchut, E. Cépa and D. Lépingle, A nonlinear SDE involving Hilbert transform. J. Funct. Anal. 165 (1999) 390-406.

[2] E. Cépa, Équations différentielles stochastiques multivoques. Sémin. Probab. XXIX (1995) 86-107.

[3] E. Cépa, Problème de Skorohod multivoque. Ann. Probab. 26 (1998) 500-532.

[4] E. Cépa and D. Lépingle, Diffusing particles with electrostatic repulsion. Probab. Theory Related Fields 107 (1997) 429-449.

[5] T. Chan, The Wigner semi-circle law and eigenvalues of matrix-valued diffusions. Probab. Theory Related Fields 93 (1992) 249-272.

[6] B. Duplantier, G.F. Lawler, J.F. Le Gall and T.J. Lyons, The geometry of Brownian curve. Bull. Sci. Math. 2 (1993) 91-106.

[7] F.J. Dyson, A Brownian motion model for the eigenvalues of a random matrix. J. Math. Phys. 3 1191-1198.

[8] W. Feller, Diffusion processes in one dimension. Trans. Amer. Math. Soc. 77 (1954) 1-31.

[9] D.J. Grabiner, Brownian motion in a Weyl chamber, non-colliding particles, and random matrices. Ann. Inst. H. Poincaré 35 (1999) 177-204. 
[10] D. Hobson and W. Werner, Non-colliding Brownian motion on the circle. Bull. London Math. Soc. 28 (1996) 643-650.

[11] I. Karatzas and S.E. Shreve, Brownian motion and stochastic calculus. Springer, Berlin Heidelberg New York (1988).

[12] P.L. Lions and A.S. Sznitman, Stochastic differential equations with reflecting boundary conditions. Comm. Pure Appl. Math. 37 (1984) 511-537.

[13] H.P. McKean, Stochastic integrals. Academic Press, New York (1969).

[14] M.L. Mehta, Random matrices. Academic Press, New York (1991).

[15] M. Metivier, Quelques problèmes liés aux systèmes infinis de particules et leurs limites. Sémin. Probab. XX (1986) $426-446$.

[16] M. Nagasawa and H. Tanaka, A diffusion process in a singular mean-drift field. Z. Wahrsch. Verw. Gebiete 68 (1985) $247-269$.

[17] R.G. Pinsky, On the convergence of diffusion processes conditioned to remain in a bounded region for large times to limiting positive recurrent diffusion processes. Ann. Probab. 13 (1985) 363-378.

[18] D. Revuz and M. Yor, Continuous martingales and Brownian motion. Springer Verlag, Berlin Heidelberg (1991).

[19] L.C.G. Rogers and Z. Shi, Interacting Brownian particles and the Wigner law. Probab. Theory Related Fields 95 (1993) 555-570.

[20] L.C.G. Rogers and D. Williams, Diffusions, Markov processes and Martingales. Wiley and Sons, New York (1987).

[21] Y. Saisho, Stochastic differential equations for multidimensional domains with reflecting boundary. Probab. Theory Related Fields 74 (1987) 455-477.

[22] H.Spohn, Dyson's model of interacting Brownian motions at arbitrary coupling strength. Markov Process. Related Fields 4 (1998) 649-661.

[23] A.S. Sznitman, Topics in propagation of chaos. École d'été Probab. Saint-Flour XIX (1991) 167-251.

[24] H. Tanaka, Stochastic differential equations with reflecting boundary conditions in convex regions. Hiroshima Math. J. 9 (1979) 163-177.

[25] D. Voiculescu, Lectures on free probability theory. École d'été Probab. Saint-Flour (1998). 